\title{
Design of Two-dimentional Code Anti-distortion on Conical Surface Based on Pre-stretching
}

\author{
Fucheng You ${ }^{1}$, Yue Cao ${ }^{1, *}$, Hechen Gong ${ }^{1}$, Chenwei Zhang ${ }^{1}$ and Liqiang Peng ${ }^{2}$ \\ ${ }^{1}$ Beijing Institute of Graphic Communication, Beijing, China \\ ${ }^{2}$ Institute of Information Engineering, Chinese Academy of Sciences, Beijing, China \\ ${ }^{*}$ Corresponding author
}

\begin{abstract}
Two-dimensional code has shown its impressive performance on mobile payment and advertising marketing. To ensure that the two-dimensional code is correctly identified by recognition devices, the traditional two-dimensional code must be attached to the surface of the plane of the object. However, if attached to the surface of conical carrier, the image of the normal two-dimensional code will be compressed into distortion because of image projection, which will lead to identify problem. To this end, we propose a novel algorithm of anti-distortion twodimensional code on conical surface based on pre-stretching, to address the challenge of identifying two-dimensional code on conical carrier. According to the spatial geometric relation of the conic, pre-stretching the normal two-dimensional code image via inverse mapping to obtain the pre-deformed two-dimensional code which will be printed and then pasted on the surface of conic. Specifically, the image of the pre-stretched two-dimensional code in the projection plane of the acquisition device is just as the normal two-dimensional code, which will be correctly identified. To better demonstrate the method, we test it on experimental simulation.
\end{abstract}

Keywords-conical; pre-stretching; two-dimensional code; projection transformation

\section{INTRODUCTION}

With the continuous development of economy and computer technology, people have higher requirements for the quality of goods, and the requirement of improving quality supervision has become the consensus of people. In the past, every kind of commodity affixed with a label, but it has been unable to meet our needs. "One thing, one code" has become an urgent needs. With the rapid development of smart phones, due to its high information content and low cost, two-dimensional code has sprang up in goods labeling. It can express information in both longitudinal and transverse directions, and has the characteristics of large storage capacity, high confidentiality, high tracking ability, strong resistance to damage, high redundancy and low cost and other characteristics [1]. The two-dimensional code expands the encoding capacity, and the scope of the code expanded to the number, letters, Chinese, pictures and sound. What's more, it can quickly correct the error code word, and the process of use of two-dimensional code could be independent of the database [2]. The application of two-dimensional code is becoming more and more abundant [3].

The two-dimensional code not only brings us convenience, but also brings a problem. In order to ensure the correct recognition of two-dimensional code, the placement of two- dimensional code is limited by certain conditions, i.e. the twodimensional code should be printed on flat or nearly flat objects, or displayed on flat screen. However, the small size commodities (such as pill bottles, injection bottles, cosmetic containers, beverage bottles, handicrafts, daily necessities, etc.) have different surface shapes and sizes, such as spherical, cylindrical, conical, and cylindrical prism combination shape, etc. Direct paste two-dimensional code on these non-flat surfaces will inevitably lead to distortion, and the conventional identification equipment (such as cell phones) can't identify the twodimensional code, which limits the scope of the application of two-dimensional code, and brings great difficulties to product quality supervision. Distorted two dimensional code is shown in Figure 1.

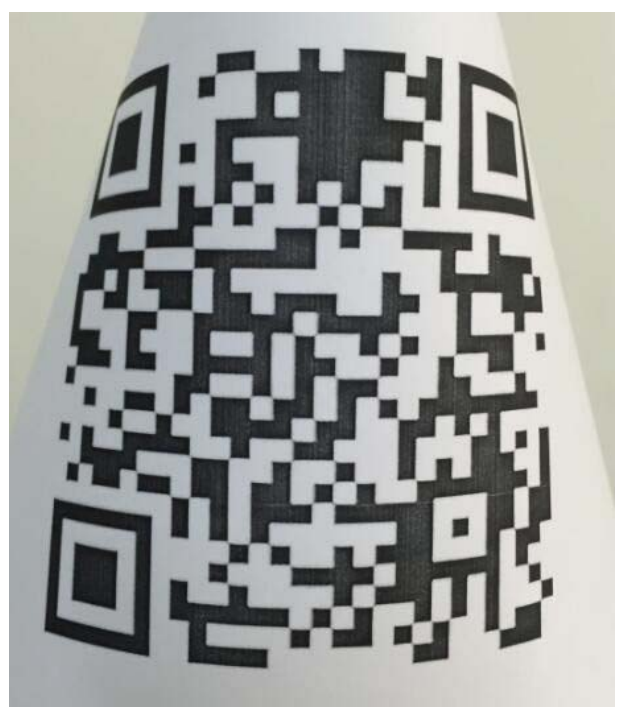

FIGURE I. DISTORTED TWO DIMENSIONAL CODE

Inspired by signal pre-stretching to counteract signal distortions in signal processing ([4] [5] [6]), we apply prestretching to two-dimensional code recognition. In this paper, we propose a novel algorithm of anti-distortion two-dimensional code on conical surface. Our algorithm based on the space geometry of two-dimensional code dependent on the carrier, used a pre-stretching algorithm to amplify or deform the normal two-dimensional code. When the distorted two-dimensional L code is pasted on the surface of a cylindrical prism combination object, when capture a two-dimensional code image using a charge coupled reading device (cell phone, etc.), the two- 
dimensional code image on the imaging projection surface is compressed into a normal two-dimensional code. Any decoding software can scan and decode at the center of the twodimensional code. Furthermore, it will improve the scope of application of two-dimensional code.

The rest paper is presented as follows. In section II, we review some related work about how to tackle the above problem. In section III, we introduce our Pre-stretching algorithm in details. And in section IV, we show the experiment design and results. Finally, section V concludes the paper.

\section{RELATED WORK}

\section{A. Three-dimensional Perspective Transformation}

In [7], it proposed a two-dimensional code recognition method based on three-dimensional perspective transformation, which can effectively identify the two-dimensional code printed on cylindrical surfaces such as beverage bottles. In this method, the key word of the image contour is detected to determine the location of the back-to-back glyph. On this basis, the key contour of the bar code is selected and Huff's transform is performed to extract the information of the perspective ellipse on the cylindrical surface. Combined with the parameters of perspective ellipse and three-dimensional perspective transformation, the transformation matrix of cylindrical noodle barcode pixels mapping directly from two-dimensional image plane to three-dimensional image space is constructed effectively and the two-dimensional code target printed on plane or cylindrical surface is reconstructed. The experimental results show that the algorithm has high accuracy for recognizing plane or cylinder two-dimensional bar codes.

\section{B. Projection Algorithm}

[8] proposed a two-dimensional barcode recognition algorithm based on projection algorithm, and studied the algorithm of 2D barcode segmentation and recognition by taking PDF417 barcode as an example. Firstly, according to the Hough transform, the barcode on the image is located, and the barcode is rotated to the horizontal using bilinear interpolation. Then the image of a single code word symbol is located in the barcode. Finally, the projection algorithm is used to identify the single code word symbol. The proposed projection algorithm can effectively remove the impact of several common image noise on bar code recognition and improve the recognition rate of bar codes. The experimental results fully show that the algorithm has good performance and meets the requirements of practical application.

\section{Correlation Matching}

Related scholars have also proposed a two-dimensional code recognition method based on correlation matching [9]. The process of the method is as follows: The improved adaptive threshold method based on surface fitting is used to segment the image. Hough transform and control point transform are used to correct the geometric distortions of the image. The template is used to match the two-dimensional code, and the sample grid is obtained by threshold the coherence coefficient. Experiments show that the algorithm can effectively improve the twodimensional code recognition efficiency and effectiveness.

\section{Correcting Parameters}

[10] proposed a method of correcting and recognizing cylinder two-dimensional codes using cylinder parameters to solve the problem of identification when the two-dimensional code was printed on a cylindrical joint of a book. When collecting the two-dimensional code printed on the cylinder surface, due to distortion of the surface, a serious distortion occurs. The acquired two-dimensional code is no longer a rectangle, result in the code can't be decoded. In this method, the three-dimensional perspective relationship is obtained by parameterizing the cylindrical model algorithm to correct the distorted two-dimensional code to obtain approximate standard two-dimensional code, and finally obtain the two-dimensional code information. Experimental results show that the algorithm has high accuracy for recognizing cylindrical two-dimensional bar codes.

\section{E. Conic Segmentation}

In [11], it proposed a two-dimensional code correction algorithm based on conic segmentation. When the twodimensional code is skewed on the cylinder and we capture a two-dimensional code image, the two-dimensional code in this image is not the expected square which presented as a rectangular two-dimensional code. The two-dimensional code becomes distorted due to a problem with the camera acquiring the image mode, which distorts the image of the original twodimensional code and Cause two-dimensional code decoding failed.

After observation of the two-dimensional code, the author found that the two-dimensional code structure is divided into horizontal and vertical straight lines that clearly divide the twodimensional image into black and white square matrices of a block. These straight lines clearly divide the two-dimensional image into blocks of black and white square matrices that are distorted and curved as they are attached to the cylinder. The basic concept of two-dimensional code image correction is to fit these curves into a conic Model, then use a conic to segment the black and white blocks of the cylinder two-dimensional code image, and then copy each of the distorted black and white blocks to the corresponding position in the blank plot. The algorithm first uses Sobel edge detection to find the edge points, sets the model of the conic curve and uses the least square error solution to do the conic curve fitting, then the fitted conic curve cuts the black and white blocks distorted in the whole twodimensional image, Finally, the corresponding position in the blank image is filled with the color of the distorted block. After several experiments, the above method can improve the recognition rate of the two-dimensional code.

In the above research works, although these algorithms have a good presentation in decoding, it is still idealized, since these methods place the correction part in the decoding stage, which results in slow decoding. To use these algorithms, the existing two-dimensional code decoding software must be modified to have complicated functions and how many types of correction functional modules are required on how many kinds of curved surfaces to be developed These kinds of function modules have a huge workload, and it is almost impossible to deploy these modules to a CCD image recognition device such as a mobile phone. 


\section{DESIGN PRINCIPLES AND CONTENT}

In everyday life, the two-dimensional code we have seen is usually printed or displayed at the approximate plane of the carrier. However, when the carrier is a cone, the twodimensional code image we collect through the image acquisition device will occur nonlinear deformation. If the twodimensional code is small compared with the conical surface, the two-dimensional code image can still be decoded by decoding software. But in other cases, the large deformation on both sides of the two-dimensional code makes the two-dimensional code can't be decoded by decoding software. Then the existing decoding software needs to be redesigned to decode it. To this end, we propose a method of design and identification of antidistortion two-dimensional code on conical surface based on pre-stretching.

\section{A. Steps of Linear Stretched Two-dimensional Code}

Figure 2 is a front view of the conical carrier. Divide the $l_{o_{1} o_{2}}$ into pixels. There are $h$ pixels between $O_{1}$ and $O_{2}$. So the conical carrier can be divided into h circles with different radius.

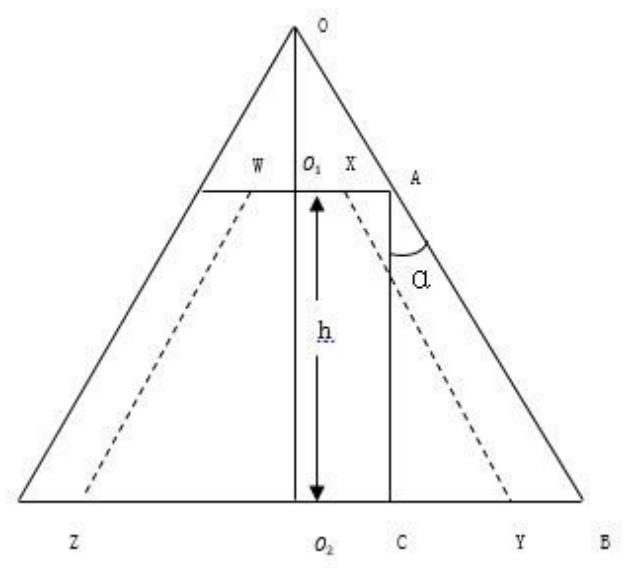

FIGURE II. FRONT VIEW OF CONICAL CARRIER

Figure 3 is one of the cross section. And the corresponding relation between the whole conical surface and its projection plane is needed. Traverse the part of linear stretched twodimensional Code in this cross section. According to the corresponding relation, we can get each pixel value from the original two-dimensional Code.

$l_{O_{1} O_{3}}$ is the projection of the $\widehat{F H}$ on the projection plane, which is the same projection in the image acquisition. $l_{O_{1} O_{3}}$ is the width of the original two-dimensional Code, and $O_{1}$ to $O_{3}$ are one row-pixels of the original two-dimensional image. The pixel value of $\mathrm{O}_{1}$ to $\mathrm{O}_{3}$ in the original two-dimensional Code is known, $\widehat{F H}$ can be calculated by the following four steps:

Step 1: Find the geometric center of the original twodimensional Code image (the symmetry axis of the original twodimensional Code image in the vertical direction). And we align it to the center of the conic section.

Step 2: According to the parameter of the conical carrier and the width of the original two-dimensional Code, we can calculate the length of $\widehat{F H}$. Then traverse the pixels of $\widehat{F H}$. According to the corresponding relation between $\widehat{\mathrm{FH}}$ and $l_{O_{1} O_{3}}$, we can get value of each pixel of $\widehat{F H}$. Corresponding relation is shown in the next step.

Step 3: Suppose $\mathrm{G}$ is an arbitrary pixel on $\widehat{F H}, \mathrm{O}_{2}$ is the corresponding pixel of the pixel $\mathrm{G}$ on the original twodimensional Code. $l_{O_{1}^{\prime} O_{2}^{\prime}}$ is the mapping segment of FG in the original two-dimensional Code.

When pixel $\mathrm{G}$ is on the left side of point $\mathrm{E}$ : arc length $l_{\widehat{F E}}$ following equation: $\widehat{F E}$ and the central angle $\beta$ (radian) have the following equation:

$$
\begin{gathered}
l_{\overparen{G E}}=l_{\widehat{F E}}-l_{\widehat{F G}} \\
\beta=l_{\overparen{G E}} / R \\
l_{O^{\prime} O_{2}^{\prime}}=\mathrm{R} \times \sin \beta \\
l_{O_{1}^{\prime} O_{2}^{\prime}}=l_{O^{\prime} O_{2}^{\prime}}-l_{O^{\prime} O_{2}^{\prime}}
\end{gathered}
$$

When point $\mathrm{G}$ is on the right side of point $E$ :

$$
\begin{gathered}
l_{\overparen{G E}}=l_{\widehat{F G}}-l_{\widehat{F E}} \\
\beta=l_{\overparen{G E}} / R \\
l_{O^{\prime} O_{2}^{\prime}}=\mathrm{R} \times \sin \beta \\
l_{O_{1}^{\prime} O_{2}^{\prime}}=l_{O^{\prime} O_{1}^{\prime}}+l_{O^{\prime} O_{2}^{\prime}}
\end{gathered}
$$

According to this, we can get the corresponding relation between the original two-dimensional Code and the linear stretched two-dimensional Code.

Step 4: Then we traverse all over the pixels on each circle that make up the pre-stretched two-dimensional Code. We can get the complete linear stretched two-dimensional Code.

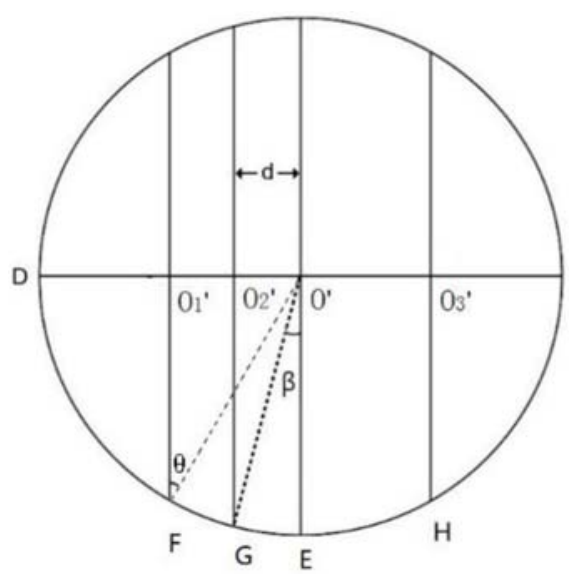

FIGURE III. CROSS SECTION OF CONICAL CARRIER

The experimental results are shown in Figure 4: 


\section{B. Steps of Nonlinear Stretched Two-dimentional Code}

Figure 5 is the expansion of the conical carrier surface. From this figure, the row pixels in linear stretching two-dimensional Code correspond to the concentric arcs of the conical carrier. We need to find the relation between the pixel-rows and concentric arcs. And get the linear stretching two-dimensional Code to make the second deformation. The nonlinear stretched twodimensional Code can be obtained after deformation.

By analyzing figure 2 and figure 5:

$$
\begin{aligned}
& l_{O^{\prime \prime} O_{1}^{\prime \prime}}=l_{O A} \\
& l_{O^{\prime \prime} O_{1}^{\prime \prime}}=l_{O B}
\end{aligned}
$$

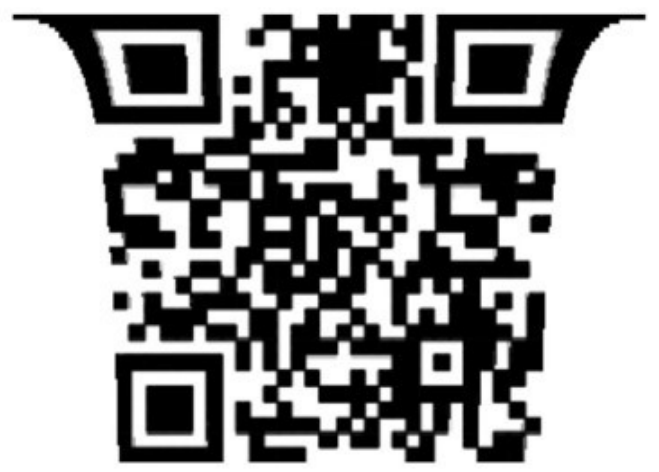

FIGURE IV. LINEAR STRETCHED TWO-DIMENTIONAL CODE

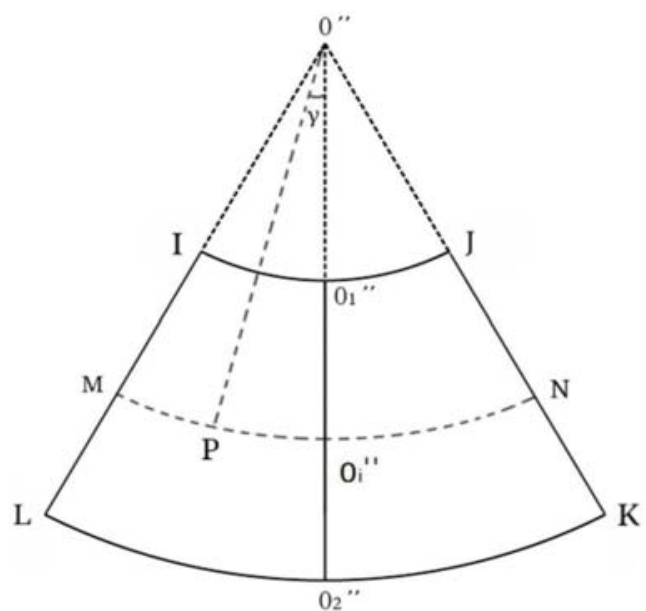

FIGURE V. EXPANSION OF CONICAL CARRIER

$\widehat{I J}$ corresponds to the first pixel-row in the linear stretched two-dimensional Code. $\widehat{L K}$ corresponds to the last pixel-row in the linear stretched two-dimensional Code. Suppose $\widehat{M N}$ be an arbitrary arc between $\widehat{I J}$ and $\widehat{L K}$, and pixel $P$ is an arbitrary pixel on $\widehat{M N}, \widehat{M N}$ corresponds to the $\mathrm{X}$-th pixel-row in the linear stretched two-dimensional Code. The corresponding pixel of the pixel $P$ on the linear stretched two-dimensional Code is the $\widehat{M P}$ th pixel in the X-th pixel-row. $\mathrm{X}$ and $\widehat{M P}$ can be obtained by the equations:

$$
\begin{gathered}
X=l_{O^{\prime \prime} O_{1}^{\prime \prime}} \times \cos \angle C A B \\
\widehat{M P}=l_{O^{\prime \prime} P} \times \angle M O^{\prime \prime} P
\end{gathered}
$$

According to the relation between the linear stretched twodimensional code and the nonlinear stretched code, the value of each pixel in the nonlinear stretching can be obtained by traversing the entire nonlinear stretched code. The experimental results are shown in Figure 6.

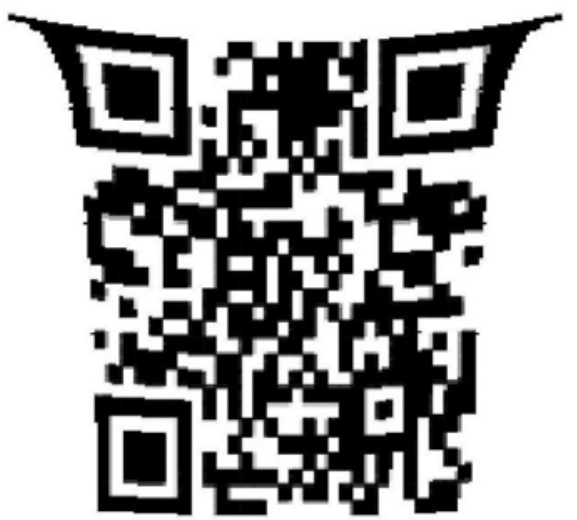

FIGURE VI. NONLINEAR STRETCHED TWO-DIMENTIONAL CODE

\section{IMPLEMENTATION AND SUMMARY}

The pre-stretching two-dimensional Code can obtain by the above algorithm as shown in Figure 6. Paste the processed picture on the conical surface as shown in figure 7. Scan the twodimensional Code image through the image acquisition device and then we can decode it easily.

In the end, the above image is identified by the decoding software and we can find out the following: When the normal two-dimensional Code print is printed on the surface of conic, the decoding device failed to identify image because the image is impressed. The pre-stretching two-dimensional Code can counteract the deformation of conical surface, so the decoding device can successfully identify image. This method of producing pre-stretching two-dimensional Code can increase the application occasions of two-dimensional Code.

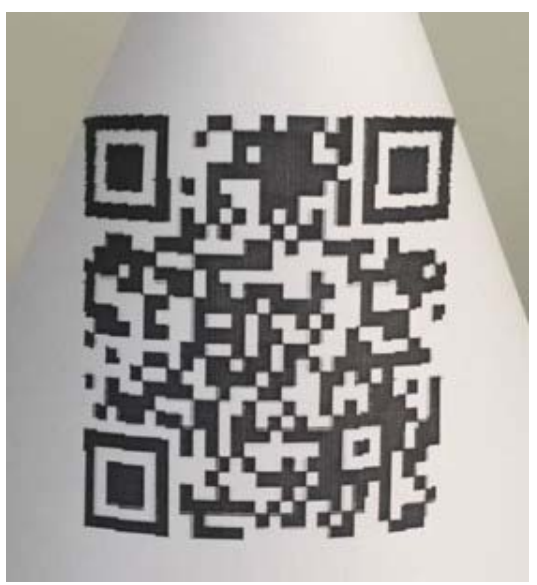

FIGURE VII. PRE-STRETCHED QR CODE ATTACHED TO CONICAL CARRIER 


\section{CONCLUSIONS}

This paper proposes a pre-stretching anti-distortion twodimensional code for conical carrier. Compared with the existing methods, our method does not need to modify the twodimensional code recognition software, and recognition is fast and more efficient. And it is a good challenge to test prestretching algorithms in more shapes.

\section{ACKNOWLEDGMENTS}

This work was partially supported by Joint Funding Project of Beijing Municipal Commission of Education and Beijing Natural Science Fund Committee (KZ201710015010), Project of National Scientific Found (No.61370188), Project of Beijing Municipal College Improvement Plan (PXM2017_014223_000063) and New Project of Green Printing and Publishing Technology by Cooperative Creating Center (PXM_014223_000025).

\section{REFERENCES}

[1] Zeng Zi Jian. Research and Implementation of TWO-DIMENTIONAL Two - dimensional Code Coding and Decoding Technology. Cheng Du: University of Electronic Science and Technology (2010).

[2] Gammer N, Cherrett T, Gutteridge C, ”Disseminating real-time bus arrival information via TWO-DIMENTIONAL code tagged bus stops: a case study of user take-up and reaction in Southampton, UK”, Journal of Transport Geography,vol.34, no. 2, (2014), pp. 254-261.

[3] Gao Feng, ”The Design and Implentation of Two-dimensional Barcode Recognition system on Mobile Phone”, Bei Jing: Beijing University of Posts and Telecommunications, (2008).

[4] Yang Youfu, Zhang Qishan, Liu Jianwei, et al. An open-loop MPM predistorter based on look-up table [J] .Beijing University of Aeronautics and Astronautics, 2012, 38 (3): 400-404.

[5] Sun Ji-li, Zhang Ping, Yang Ruliang. Pre-Distortion Compensation of On-orbit SARLFM Signals [J]. Journal of Harbin Institute of Technology, 2012, 44 (3): 137-143.

[6] Tang Chengkai, Lian Baowang, Zhang Lingling. Recalling pre-distortion algorithm with memory under 16APSK modulation in satellite communication $[\mathrm{J}]$.Journal of Northwestern Polytechnical University, 2014 (6): 962-966.

[7] Yang $\mathrm{Xu}$, (2013). Research and Implementation of Distorted TwoDimensional Code Recognition Algorithm (Doctoral dissertation, Fudan University).

[8] LIU Ning-Zhong, YANG Jing-Yu. Dimensional Bar Code Recognition Based on Projection Algorithm [J] .Computer Engineering, 2002, 28 (9): 32-33.

[9] XIONG Yong, WANG Lu-Cai, AI Qiong-Long.Identification of TWODIMENTIONAL Code Based on Correlation Matching [J]. Chinese Journal of Instrumentation, 2011, 32 (3): 571-576.

[10] Lay K T, Wang L J, Wang C H. Rectification of TWO-DIMENTIONALcode images using the parametric cylindrical surface model[C]// International Symposium on Next-Generation Electronics. IEEE, 2015:1-5.

[11] Kuen-Tsair Lay, Lee-Jyi Wang, Pei-Lun Han, et al. Rectification of images of TWO-DIMENTIONAL codes posted on cylinders by conic segmentation[C]// IEEE International Conference on Signal and Image Processing Applications. IEEE, 2015. 